\title{
Border-Flow Control by means of Phase Shifting Transformers
}

\author{
J. Verboomen, Member IEEE, D. Van Hertem, Graduate Student Member IEEE, P. H. Schavemaker, \\ W. L. Kling, Member IEEE, R. Belmans, Fellow IEEE
}

\begin{abstract}
In this paper, a control scheme is demonstrated that regulates multiple Phase Shifting Transformers (PSTs) to equally load the interconnectors of a border. A crucial step in the development of the control scheme is the derivation of Phase Shifter Distribution Factors (PSDFs), which indicate the influence of a PST on the active power flow on a certain line. Based on these PSDFs, the Linear Least Squares (LLS) method is used to calculate the optimal PST settings. The degree to which an even repartition can be obtained, depends on the number of PSTs in relation to the number of interconnectors. As a casestudy, simulations are performed involving the Dutch and Belgian interconnectors.
\end{abstract}

Index Terms - Phase Shifting Transformer, Load Flow Control, Linear Least Squares

\section{INTRODUCTION}

D UE to uneven loading of interconnectors in meshed networks, the total cross-border capacity available for import and export of electrical energy, is lower than might be expected when looking at the capacities of the tie lines. To improve the situation, the Dutch Transmission System Operator (TSO) installed two phase shifting transformers (PSTs)[1] at the Meeden substation in the north of the Netherlands [2, 3]. The southern part of the country is closer to the centre of the meshed European grid than the northern part, which leads to congestion problems on the southern interconnectors with Germany. The PSTs can divert power to the northern interconnector, loading the lines more evenly.

To cope with the growing problem of transit flows, the Belgian Transmission System Operator has decided to install several PSTs, because a single device can only shift power to other lines but can not fully control it. The plans are to install one device in Zandvliet and two in the Van Eyck substation on the Belgian-Dutch border. Another PST at the Gronau substation in Germany has been functioning already for a long time.

The use of several PSTs in a relatively small geographic area must be treated carefully, as a poor coordination can lead to inefficient use of infrastructure or even to situations where the security of supply is no longer guaranteed. The goal of this paper is to study how the PSTs (once the Belgian PSTs are installed) can be controlled in order to obtain an optimal or near-optimal exchange situation for both the Netherlands and

J.Verboomen, P.H.Schavemaker and W.L.Kling are with the Electrical Power Systems Group of the Delft University of Technology, Mekelweg 4, 2628 CD Delft, The Netherlands, Email: j.verboomen@tudelft.nl, p.h.schavemaker@tudelft.nl,w.l.kling@tudelft.nl

D.Van Hertem and R.Belmans are with the ELECTA research group of the K.U.Leuven,Belgium.
Belgium. The solution can be found by optimising all PSTs as described in [4], or by controlling the Dutch-German and the Belgian-Dutch border separately in such a way that the loadings of the tie lines of that border are even, or at least as even as possible.

The authors stress that the developed control scheme is only one possible approach to PST coordination. At this point, there is no general agreement between TSOs on equal power repartition, or on any coordinated use of PST control.

In section II, DC power flow equations are developed accounting for phase shifters. Then, section III explains the principles of linear least squares approximation. These two concepts are combined in a single border flow control algorithm in section IV. A combined control scheme for multiple borders is proposed in section V. The effect of topology changes on this control scheme is discussed in section VI. Finally, section VII shows a more detailed modelling of the phase shifters.

\section{DC POWER Flow EQUATIONS}

By using a DC load flow approximation $[5,6]$, the power $P_{i j}$ through a transmission line with a PST and the power $P_{p q}$ through a line that is influenced by a PST somewhere else in the system can be written as:

$$
\begin{gathered}
P_{i j}=P_{i j}^{\dagger}+\alpha_{i j} \xi_{\alpha}^{i j} \\
P_{p q}=P_{p q}^{\dagger}+\alpha_{i j} \xi_{\alpha}^{p q}
\end{gathered}
$$

where $\alpha_{i j}$ is the phase shift angle of PST $i j$ and $P_{i j}^{\dagger}$ and $P_{p q}^{\dagger}$ are the line power flows at zero phase shift.

The derivative of the power through the line to $\alpha$ can be referred to as the Phase Shifter Distribution Factor (PSDF) [8], and can be expressed as follows:

$$
\begin{aligned}
& \xi_{\alpha}^{i j}=\frac{\partial P_{i j}}{\partial \alpha}=y_{i j}\left(1+y_{i j}\left(2 c_{i j}-c_{i i}-c_{j j}\right)\right) \\
& \xi_{\alpha}^{p q}=\frac{\partial P_{p q}}{\partial \alpha}=y_{p q} y_{i j}\left(c_{p j}-c_{p i}+c_{q i}-c_{q j}\right)
\end{aligned}
$$

where $y_{i j}$ and $c_{i j}$ indicate the element $i j$ of the admittance matrix and inverse admittance matrix respectively.

If multiple PSTs are installed in the system, the equations for the line power flow must be generalised. The power flow through a line with a PST and that is influenced by other PSTs in lines $(m, n)$ is: 
TABLE I

$$
P_{i j}=P_{i j}^{\dagger}+\alpha_{i j} \xi_{\alpha_{i j}}^{i j}+\sum_{\substack{(m, n) \\(m, n) \neq(i, j)}} \alpha_{m n} \xi_{\alpha_{m n}}^{i j}
$$

It can be seen that every PST contributes an extra term to the equation. The power flow through the line is now a linear function of the different phase shifter settings.

The power flow through a line without a PST, influenced by PSTs in lines $(m, n)$, is:

$$
P_{p q}=P_{p q}^{\dagger}+\sum_{(m, n)} \alpha_{m n} \xi_{\alpha_{m n}}^{p q}
$$

\section{LINEAR LEAST SQUARES}

Suppose the following overdetermined system of linear equations is given:

$$
A x \approx b
$$

In the Linear Least Squares (LLS) approach [9], the aim is to find the $\boldsymbol{x}$ for which $\|\boldsymbol{A} \boldsymbol{x}-\boldsymbol{b}\|^{2}$ is minimal (hence the name). This can be written as:

$$
\min _{\boldsymbol{x}} J=(\boldsymbol{A} \boldsymbol{x}-\boldsymbol{b})^{T}(\boldsymbol{A} \boldsymbol{x}-\boldsymbol{b})
$$

It is pretty straightforward to verify that this minimisation problem has the following solution:

$$
\boldsymbol{x}_{0}=\left(\boldsymbol{A}^{T} \boldsymbol{A}\right)^{-1} \boldsymbol{A}^{T} \boldsymbol{b}
$$

If a border with all its interconnectors is considered, then the active power flows can be described by the following matrix equation:

$$
\boldsymbol{P}=\boldsymbol{P}^{\dagger}+\boldsymbol{\Xi} \cdot \boldsymbol{\Delta} \boldsymbol{\alpha} \approx \boldsymbol{P}_{r e f}
$$

where $\boldsymbol{P}^{\dagger}$ is the vector of power flows with the PSTs set to their reference position (for example: all at zero degrees), $\boldsymbol{\Xi}$ is a matrix of PSDFs and $\boldsymbol{P}_{r e f}$ is a reference power distribution which is to be approximated. In the border balancing problem, the relative loading of the interconnectors should be made equal. So, the elements of $P_{r e f}$ are:

$$
\frac{P_{i}}{P_{i, r}}=\frac{\sum_{i=1}^{n} P_{i}}{\sum_{i=1}^{n} P_{i, r}} \Longleftrightarrow P_{i}=\frac{\sum_{i=1}^{n} P_{i}}{\sum_{i=1}^{n} P_{i, r}} \cdot P_{i, r}
$$

where $P_{i}$ is the power flow through one of the interconnectors and $P_{i, r}$ is the rated power of that line. If (10) is rearranged, it can be written as:

$$
\Xi \cdot \Delta \boldsymbol{\Delta} \approx \boldsymbol{P}_{r e f}-\boldsymbol{P}^{\dagger}
$$

which can be identified with (7). The change in phase shifter settings that results in the best approximation of an equal loading scenario can be found by using (9).
Calculated AND Simulated Line loadings at the Dutch-German BORDER FOR 4 CASES

\begin{tabular}{|r|cccc|}
\hline & $\begin{array}{c}L_{1} \\
(\mathrm{pu})\end{array}$ & $\begin{array}{c}L_{2} \\
(\mathrm{pu})\end{array}$ & $\begin{array}{c}L_{3} \\
(\mathrm{pu})\end{array}$ & $\begin{array}{c}L_{4} \\
(\mathrm{pu})\end{array}$ \\
\hline \hline base 1 & 0.15 & 0.53 & 0.48 & 0.57 \\
calculated optimum 1 & 0.43 & 0.43 & 0.39 & 0.49 \\
simulated optimum 1 & 0.47 & 0.42 & 0.38 & 0.48 \\
\hline \hline base 2 & 0.20 & 0.58 & 0.44 & 0.54 \\
calculated optimum 2 & 0.43 & 0.43 & 0.39 & 0.48 \\
simulated optimum 2 & 0.47 & 0.41 & 0.38 & 0.48 \\
\hline \hline base 3 & 0.20 & 0.54 & 0.24 & 0.29 \\
calculated optimum 3 & 0.30 & 0.30 & 0.27 & 0.32 \\
simulated optimum 3 & 0.31 & 0.26 & 0.28 & 0.32 \\
\hline \hline base 4 & 0.10 & 0.38 & 0.16 & 0.17 \\
calculated optimum 4 & 0.19 & 0.19 & 0.19 & 0.19 \\
simulated optimum 4 & 0.20 & 0.16 & 0.19 & 0.20 \\
\hline
\end{tabular}

TABLE II

CAlculated AND Simulated line loAdings at THE DUtCh-Belgian BORDER FOR 4 CASES

\begin{tabular}{|l|c|ccc|}
\hline \multicolumn{2}{|c|}{ case } & $\begin{array}{c}L_{1} \\
(\mathrm{pu})\end{array}$ & $\begin{array}{c}L_{2} \\
(\mathrm{pu})\end{array}$ & $\begin{array}{c}L_{3} \\
(\mathrm{pu})\end{array}$ \\
\hline \multirow{4}{*}{ Base case } & 1 & 0.12 & -0.11 & 0.48 \\
& 2 & 0.10 & -0.10 & 0.48 \\
& 3 & 0.15 & -0.11 & 0.48 \\
\hline \multirow{5}{*}{ Simulated optimum } & 4 & 0.23 & -0.09 & 0.52 \\
\hline \multirow{5}{*}{} & $1 / 0.07$ & 0.07 & 0.09 & 0.02 \\
& $1 / 0.14$ & 0.15 & 0.17 & 0.10 \\
& $1 / 0.21$ & 0.22 & 0.24 & 0.18 \\
\cline { 2 - 5 } & $2 / 0.07$ & 0.07 & 0.09 & 0.02 \\
& $2 / 0.14$ & 0.15 & 0.16 & 0.10 \\
\cline { 2 - 5 } & $2 / 0.21$ & 0.23 & 0.24 & 0.19 \\
\cline { 2 - 5 } & $3 / 0.07$ & 0.06 & 0.09 & 0.02 \\
& $3 / 0.14$ & 0.14 & 0.17 & 0.10 \\
& $3 / 0.21$ & 0.22 & 0.24 & 0.18 \\
\cline { 2 - 5 } & $4 / 0.07$ & 0.05 & 0.09 & 0.02 \\
& $4 / 0.14$ & 0.13 & 0.16 & 0.10 \\
& $4 / 0.21$ & 0.21 & 0.24 & 0.18 \\
\hline
\end{tabular}

\section{Single Border CONTROL}

\section{A. Border Types}

Borders can be classified as one of the following types:

- Type 1 borders have less than $l-1$ PSTs for $l$ interconnectors. This means that the flow distribution on the border can not be fully controlled.

- Type 2 borders have $l-1$ PSTs for $l$ interconnectors. In this case, the flow distribution can be controlled, but the total transfer can not be fixed.

- Type 3 borders have a PST in every interconnector. In this way, the flow distribution can be controlled, but also the total transfer over the border can be set.

\section{B. Dutch-German Border}

The Dutch-German border is of type 1. The LLS method is applied starting from four base cases, in which the settings of the PSTs on the Belgian-Dutch border are different and the PSTs on the Dutch-German border are at zero phase shift. The two devices in Meeden are considered as one, because they are operated in that way. The PST of Gronau in Germany is also taken into account. For every case, the optimal $\Delta \alpha$ is 
calculated for Meeden and Gronau. The results can be seen in Table I.

The optimum PST settings and the corresponding line loadings are calculated by LLS and equations (5) and (6). The optimum settings were applied in a full AC simulation model in PSS/E in order to verify the line loadings ("simulated optimum" in the table).

From the results, the following conclusions can be drawn:

- The LLS method is able to balance the border flows to a certain extent. However, perfect balancing is not possible because four line flows can not be fully controlled by only two PSTs.

- The calculated and simulated results are slightly different due to the limitations of the DC load flow approach.

\section{Dutch-Belgian Border}

The Dutch-Belgian border will be of type 3 after the PSTs in Belgium are installed. An LLS approach is not strictly necessary because the desired line flows can be obtained exactly, since there are 3 flows to control with 3 PSTs (i.e a square system of equations). In this case, it suffices to solve the set of linear equations. If the aim is an equal loading, then the desired line powers are set to the desired power transfer divided by three (since the rated powers of the lines at the Dutch-Belgian border are all equal).

A calculation is performed for four different cases, which differ by the settings of the PSTs at the German-Dutch border. In the base cases, the PSTs on the Dutch-Belgian border are at zero phase shift. In every optimised case the desired power transfer from Belgium to the Netherlands is set to 300, 600 and $900 \mathrm{MW}$ successively. For these three transfers, the line loadings should be $0.07,0.14$ and 0.21 respectively. The calculated PST settings to obtain an equal loading as well as to establish the desired transfer are calculated and used in the simulation model. The resulting line loadings from the simulation can be seen in Table II. The results from the simulations can sometimes differ considerably from the desired values. The main cause can be found in the limitations of the DC load flow approach.

\section{COMBInEd Border CONTROL}

The two LLS schemes are combined in one control algorithm, which is drawn in Fig. 1. $P_{\text {init }}\left(P^{\dagger}\right.$ in eq. 10) is a vector with system flows that correspond to the initial phase shifter settings. The LLS blocks solve eq. 10; note that the Belgian-Dutch border has an extra input for the total power exchange $\left(P_{r e f}\right)$. The initial line powers are applied to the LLS functions, resulting in a $\Delta \alpha$ vector for each border. The PST is modelled as a combination of a sign function, a gain and an integrator. The integrator and sign function result in a linear ramping behaviour, which approximates the behaviour of the mechanical tap changer. The gain $K$ determines the slope of the ramp (i.e. the speed of the tap changer).

The settings of the PSTs are fed into a system block. In this block, a multiplication with the PSDF matrix is performed.

Fig. 2 shows a simulation example. At time $t=0$, all PSTs have a zero degree setting. The initial reference transfer

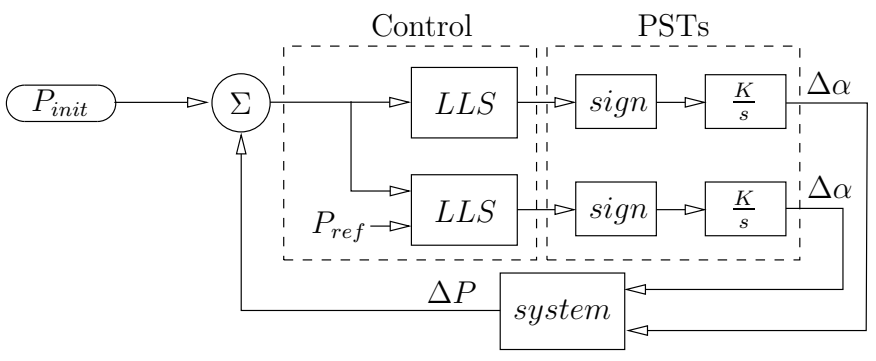

Fig. 1. Block diagram for the combined border control

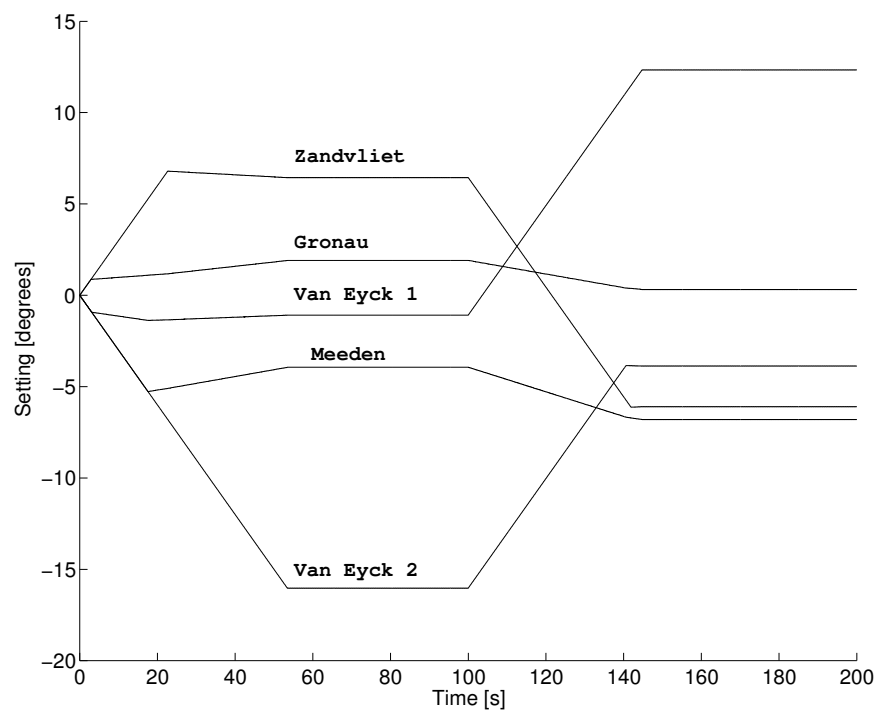

(a) PST settings

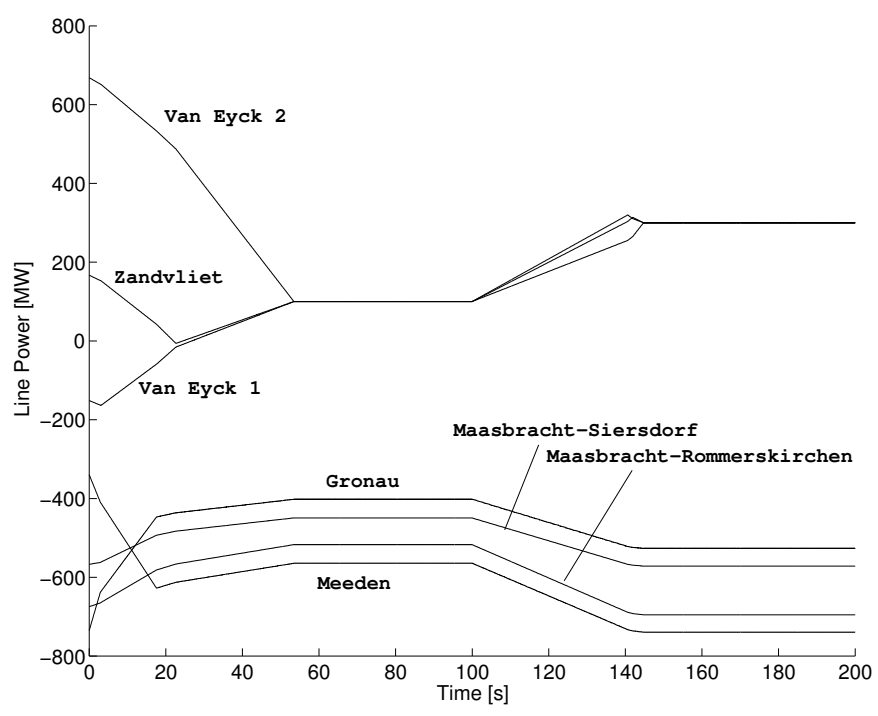

(b) Line powers

Fig. 2. PST settings and line powers for a test case. The reference power exchange between Belgium and the Netherlands is $300 \mathrm{MW}$ at first, and steps to $900 \mathrm{MW}$ at $t=100 \mathrm{~s}$.

between Belgium and the Netherlands is set to $300 \mathrm{MW}$. Since the Belgian-Dutch interconnectors all have equal nominal powers, the flows on this border converge towards $100 \mathrm{MW}$. At $t=100 \mathrm{~s}$, the reference power is altered to $900 \mathrm{MW}$. Although the LLS calculations are virtually instantaneous, a 


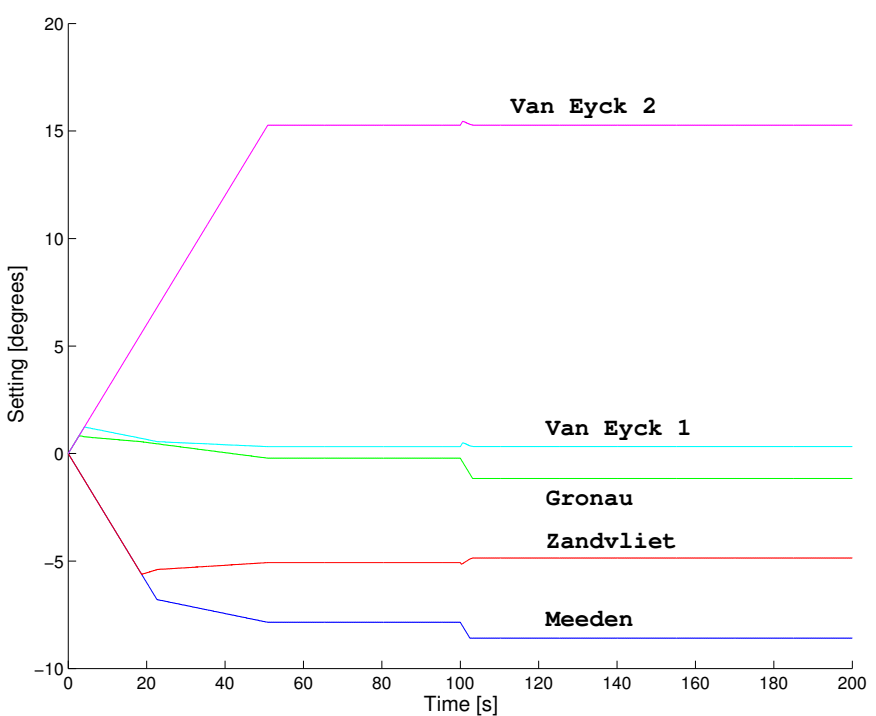

(a) PST settings

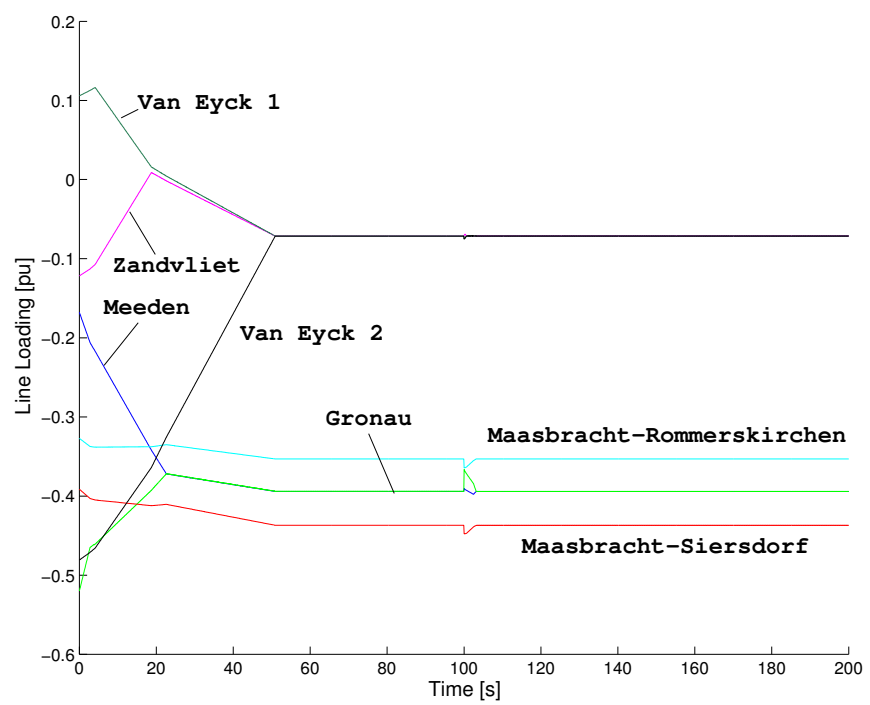

(b) Line loadings

Fig. 3. PST settings and line loadings for the Benelux case. At $t=100 \mathrm{~s}$, a line from Hengelo to Doetinchem is tripped. The LLS controllers are updated instantaneously.

delay is introduced by the mechanical tap changers of the PSTs.

\section{INFLUENCE OF GRID TOPOLOGY CHANGES}

The LLS controller relies on the PSDF matrix. If the system topology changes, for instance due to a line outage, this matrix must be updated. If the PSDF matrix in the LLS blocks is not updated, errors can occur. In the following sections, a contingency is simulated by changing the PSDF matrix in the system block in Fig. 1 and updating the $P_{\text {init }}$ vector to new values.

\section{A. internal contingencies}

As an example of an internal contingency, a $380 \mathrm{kV}$ line between Hengelo and Doetinchem is taken out of service

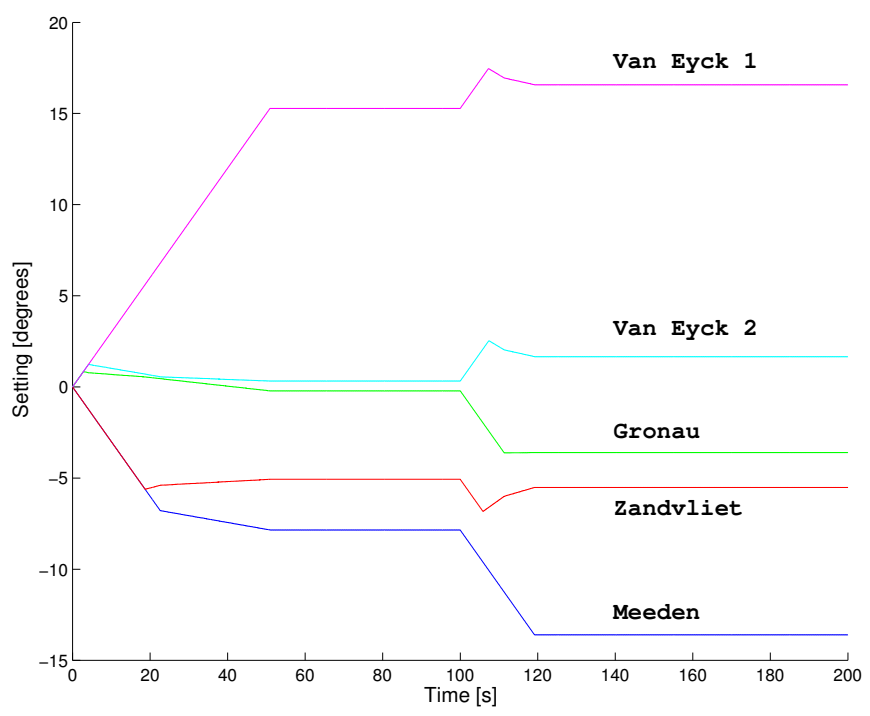

(a) PST settings

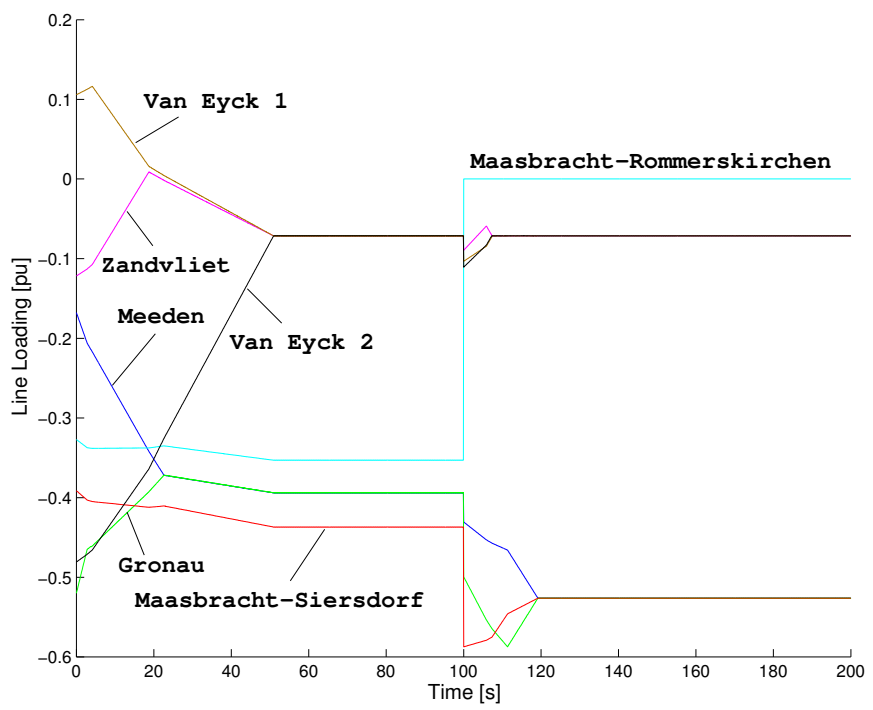

(b) Line loadings

Fig. 4. PST settings and line loadings for the Benelux case. At $t=100 \mathrm{~s}$, the line from Maasbracht to Rommerskirchen is tripped. The LLS controllers are updated instantaneously.

at $t=100 \mathrm{~s}$. The PSDF matrix in the LLS blocks is instantaneously updated. The results can be seen in Fig. 3 . After the contingency, the controller adapts the PST settings in order to return to the optimal line loadings. If the PSDF matrix in the LLS blocks is not updated in this case, there is no significant difference with the situation where they are updated. The reason for this is the minor impact of an internal contingency on the PSDF matrix.

\section{B. interconnector contingencies}

If a contingency occurs on an interconnector, the change in the PSDF matrix is much larger. If the PSDF matrix in the LLS blocks is not updated when this kind of outage occurs, the errors can be large. As an example, a simulation is performed in which the line Maasbracht-Rommerskirchen is tripped at $t=100 \mathrm{~s}$. From the simulation results in Fig. 4, we can 


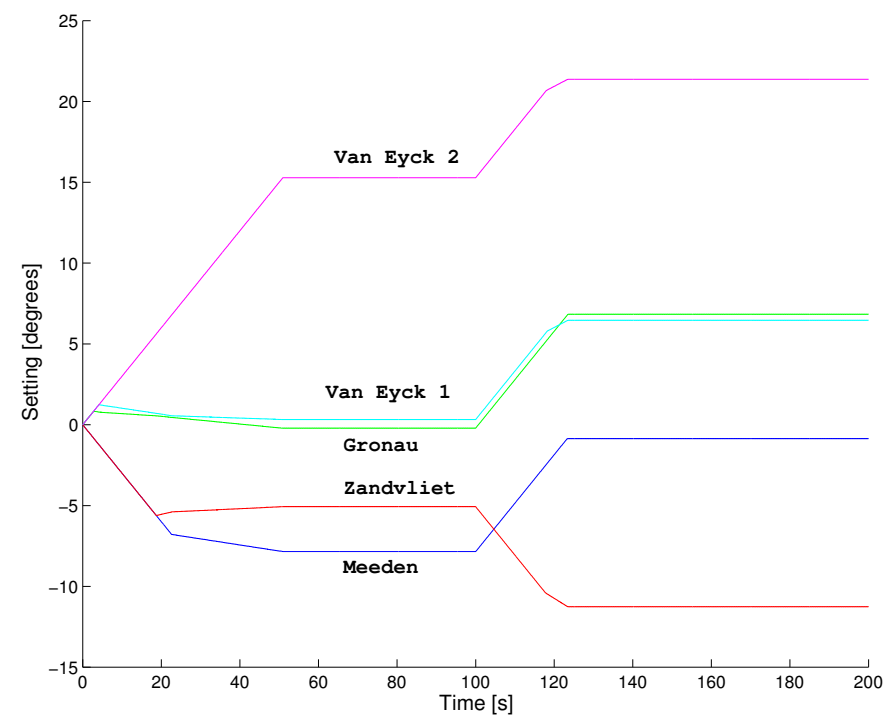

(a) PST settings

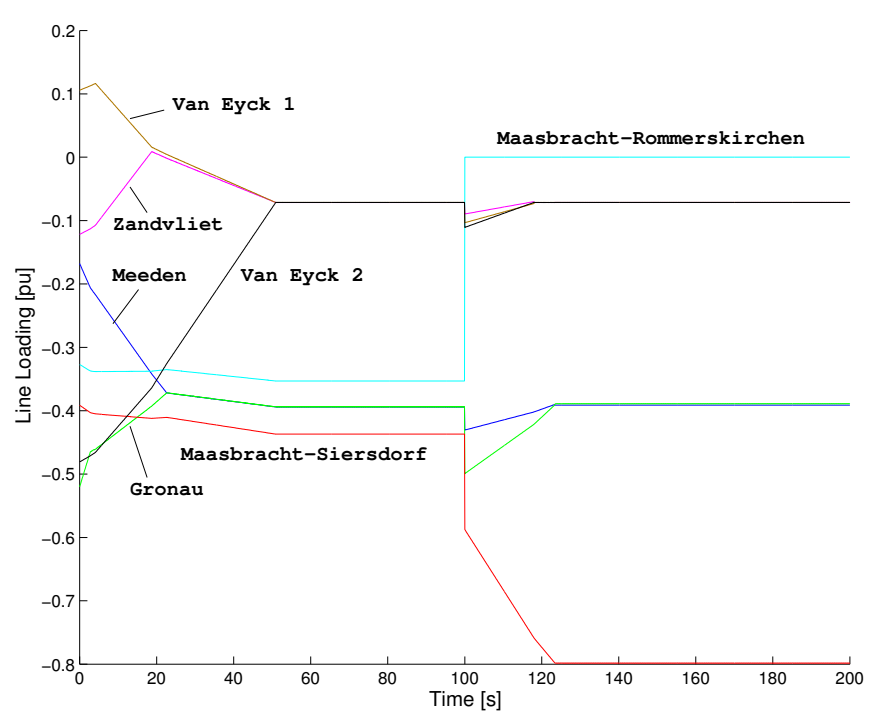

(b) Line loadings

Fig. 5. PST settings and line loadings for the Benelux case. At $t=100 \mathrm{~s}$, the line from Maasbracht to Rommerskirchen is tripped. The LLS controllers are not updated

see that the outage is counteracted by PST control actions. The Dutch-German border becomes a type 2 border after the contingency, so that the loading of the remaining lines can be made exactly equal.

If the PSDF matrix in the LLS blocks is not updated, a large error is introduced, as shown in Fig. 5. The same setpoint for every line power is maintained, so the Meeden and Gronau lines carry the same power as before. Because the line to Rommerkirchen is out of service, its complete power flow is now transferred to the line to Siersdorf.

\section{Detailed Modelling OF PST}

In the previous sections, it was assumed that the PST setting can be changed in a continuous way. This is however not the case in practice due to the mechanical tap changer. This discrete behaviour can be simulated by adding a quantiser

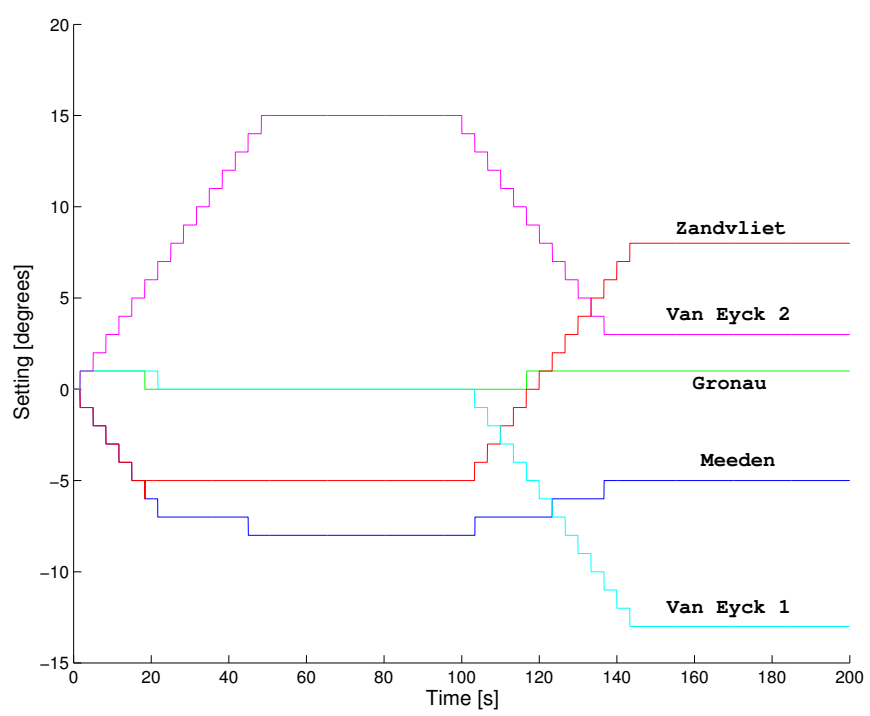

(a) PST settings

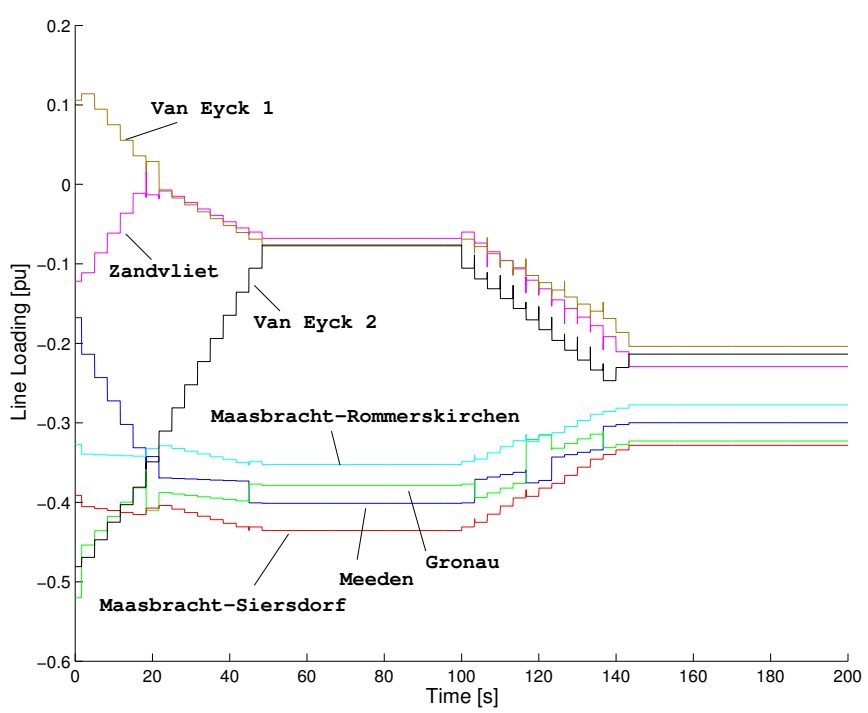

(b) Line loadings

Fig. 6. PST settings and line loadings for a test case with discrete modelling. The reference power exchange between Belgium and the Netherlands is 300 MW at first, and steps to $900 \mathrm{MW}$ at $t=100 \mathrm{~s}$.

block after the integrator in the PST model. There are several consequences to this:

- As the PSTs can only be set to discrete positions, the power flows over the lines can be controlled in a discrete way. The loadings of the interconnectors can not be made exactly equal in the general case due to this fact.

- Small disturbances and noise can result in constant switching of a PST between two states. In order to avoid this problem, a dead band block is inserted after the LLS control block.

Simulation results can be seen in Fig. 6 .

\section{CONCLUSION}

In this paper a control scheme for multiple phase shifters installed in cross-border tie lines is presented. Depending on the number of phase shifters in relation to the number of tie 
lines, the total transfer can be controlled and/or the power distribution over the lines can be altered. These principles are demonstrated for the Dutch-German and the Dutch-Belgian border. The simulations show that in some cases, deviations can occur due to the DC load flow approximations that are used. It is shown that if a contingency occurs, an update of the controller parameters can be crucial.

\section{ACKNOWLEDGMENT}

This research at the TU Delft has been performed within the framework of the research program 'intelligent power systems' that is supported financially by SenterNovem. SenterNovem is an agency of the Dutch ministry of Economic Affairs. The research performed at the KU Leuven is financially supported by the Belgian 'Fonds voor Wetenschappelijk Onderzoek (F.W.O.)-Vlaanderen'. Dirk Van Hertem is a doctoral research assistant of the F.W.O.-Vlaanderen.

\section{REFERENCES}

[1] IEEE Power Engineering Society, "C57.135 : IEEE Guide for the Application, Specification, and Testing of Phase-Shifting Transformers," May 2002.

[2] W. L. Kling, D. A. M. Klaar, J. H. Schuld, A. J. L. M. Kanters, C. G. A. Koreman, H. F. Reijnders, and C. J. G. Spoorenberg, "Phase shifting transformers installed in the Netherlands in order to increase available international transmission capacity," in CIGRE Session 2004 - C2-207, 2004.

[3] C. J. G. Spoorenberg, B. F. van Hulst, and H. F. Reijnders, "Specific aspects of design and testing of a phase shifting transformer," in XIIIth International Symposium on High Voltage Engineering, 2003.

[4] J. Verboomen, D. Van Hertem, P. H. Schavemaker, F. J. C. M. Spaan, J.-M. Delincé, W. L. Kling, and R. Belmans, "Monte carlo simulation techniques for optimisation of phase shifter settings," European Transactions on Electrical Power, 2007, accepted for publication.

[5] M. Ilić and J. Zaborsky, Dynamics and Control of Large Electric Power Systems. John Wiley \& Sons, 2000.

[6] D. Van Hertem, J. Verboomen, K. Purchala, R. Belmans, and W. L. Kling, "Usefulness of DC Power Flow for Active Power Flow Analysis with Flow Controlling Devices," in IEE International Conference on AC and DC Power Transmission 2006, London, United Kingdom, March 2006.

[7] J. Verboomen, D. Van Hertem, P. H. Schavemaker, W. L. Kling, and R. Belmans, "An analytical approach to grid operation by using phase shifting transformers," IEEE Proceedings on Power Systems, 2007, to be submitted.

[8] Z. X. Han, "Phase Shifter and Power Flow Control," IEEE Transactions on Power Apparatus and Systems, vol. PAS-101, no. 10, pp. 3790-3795, October 1982.

[9] P. E. Gill, W. Murray, and M. H. Wright, Practical Optimization. Acadmic Press, 1981.

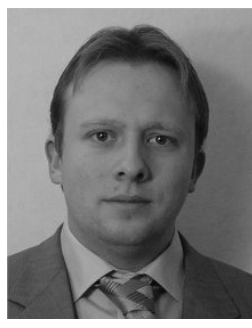

Jody Verboomen obtained his Master in Industria Sciences (Electronics) from Groep T Hogeschool in Leuven, Belgium in 2001. He obtained his M.Sc. in Electrical Engineering from the Catholic University of Leuven (KUL), Belgium in 2004. He is currently working towards a Ph.D. on the application of FACTS and phase shifters in transmission systems in the Electrical Power System (EPS) laboratory of the Delft University of Technology, The Netherlands. His research is funded by SenterNovem, an agency of the Dutch ministry of Economical Affairs.

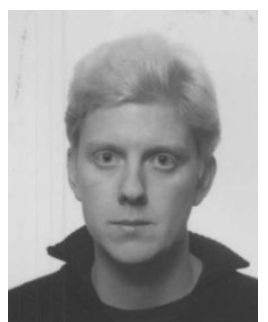

Dirk Van Hertem graduated as a M.Eng. in 2001 from the KHK, Geel/Belgium and as a M.Sc. in Electrical Engineering from the KUL, Leuven/Belgium in 2003. From 2003 he has been working towards a Ph.D. in the ELECTA research group, department of Electrical Engineering of the K.U.Leuven, Belgium. From October 2004, he is a research assistant for the F.W.O.-Vl. In 2001, his masters thesis received the 'V.I.K. award' and in 2004, he received the 'K.B.V.E. R\&D award' for his second masters thesis. His special fields of interest are power system control and optimization, including power flow controlling devices.

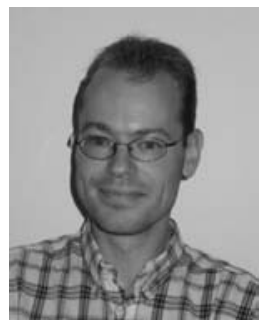

Pieter H. Schavemaker obtained his M.Sc. in Electrical Engineering from the Delft University of Technology in 1994 and he obtained his Ph.D. in Electrical Engineering from the Delft University of Technology in 2002. Since 1996 he has been with the Power Systems Laboratory where he is currently Assistant Professor. His main research interests include power system transients and power system calculations.

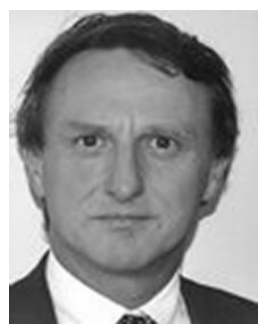

Wil L. Kling received his M.S-degree in electrical engineering from the Technical University of Eindhoven in 1978. Since 1993 he has been a (parttime) professor with the Department of Electrical Engineering at Delft University of Technology, in the field of Power Systems Engineering. In addition, he is with the Network Operations department of TenneT (the Dutch Transmission System Operator). Since 2000, he has also been a part-time professor at the TU Eindhoven. His area of interest is related to planning and operations of power systems. He is the project leader of the research programme 'Intelligent Power Systems', sponsored by SenterNovem, an agency of the Dutch Ministry of Economic Affairs. Prof. Kling is involved in scientific organizations such as CIGRE and the IEEE. As Netherlands' representative, he is a member of CIGRE Study Committee C6 Distribution Systems and Dispersed Generation, and the Administrative Council of CIGRE. Furthermore, he is involved in several international working groups in the field of network planning and system studies, within UCTE, Eurelectric and other bodies.

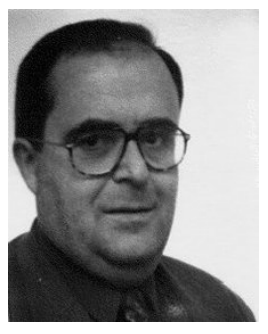

Ronnie Belmans received the M.S. degree in electrical engineering in 1979, the Ph.D. in 1984, and the Special Doctorate in 1989 from the K.U.Leuven, Belgium and the Habilitierung from the RWTH, Aachen, Germany, in 1993. Currently, he is full professor with K.U.Leuven, teaching electrical machines and variable speed drives. He is appointed visiting professor at Imperial College in London. $\mathrm{He}$ is also President of UIE. He was with the Laboratory for Electrical Machines of the RWTH, Aachen, Germany (Von Humboldt Fellow, Oct.' 88 Sept.' 89). Oct.' 89-Sept.'90, he was visiting associate professor at Mc Master University, Hamilton, Ont., Canada. During the academic year 1995-1996 he occupied the Chair at the London University, offered by the Anglo-Belgian Society. Dr. Belmans is a fellow of both the IEEE and the IEE (United Kingdom). He is the chairman of the board of Elia, the Belgian transmission grid operator. 